\title{
The effects of iron deficiency on the level of oxidative stress and antioxidant defenses in suckling piglets
}

\author{
Jonáš Vaňhara ${ }^{1}, Z_{\text {deněk Fajt }}{ }^{1}$, Jana Blahová2 ${ }^{2}$ Veronika Tykačová3, Karolína Píšt'ková3, \\ Martin Hostovský2 ${ }^{2}$ Zdenka Kazatelová ${ }^{1}$, Jan Vašek ${ }^{1}$, Martin Svoboda ${ }^{1}$ \\ University of Veterinary and Pharmaceutical Sciences Brno, ${ }^{1}$ Faculty of Veterinary Medicine, Ruminant and \\ Swine Clinic, ${ }^{2}$ Faculty of Veterinary Hygiene and Ecology, Department of Animal Welfare, Protection and \\ Behaviour, ${ }^{3}$ Faculty of Veterinary Medicine, Large Animal Clinical Laboratory, Brno, Czech Republic
}

Received October 13, 2017

Accepted December 19, 2017

\begin{abstract}
The aim of the study was to evaluate the impact of iron deficiency on the oxidative stress and antioxidant defenses in suckling piglets. Piglets in the experimental group were given no iron supplement till the age of 21 days. Piglets in the control group were injected i.m. with gleptoferronum at the age of 3 days. Blood samples were taken at $3,21,28$, and 35 days of age and examined for haematological and biochemical indices. Iron deficiency in the experimental group resulted in the development of anaemia. Significantly lower ceruloplasmin activities in blood plasma were found in the anaemic piglets. The other biochemical indices of the oxidative status (thiobarbituric acid reacting substances, carbonyl proteins, super oxide dismutase, glutathione peroxidase, trolox equivalent antioxidant capacity) were comparable between the experimental and the control group. It can be concluded that apart from lower ceruloplasmin activities the oxidative status of piglets was not affected negatively by iron deficiency.
\end{abstract}

Anaemia, haematology, ceruloplasmin, swine, carbonyl proteins

Iron is an important mineral for swine. Its deficiency has caused serious problems in pig production. The iron requirements of suckling piglets cannot be covered by the foetal reserves only. This results in iron deficiency and the development of anaemia in the first days of life (Venn et al. 1947). Most of the total body iron is found in haemoglobin (HGB), which is responsible for oxygen transport. The cellular energy metabolism is dependent on oxygen, therefore, anaemia can have a major impact on the organism (Nagababu et al. 2008).

Apart from direct effects on haemoglobin synthesis, iron deficiency can affect the function of many enzymes containing iron $(\mathrm{Fe})$ such as cytochromes, catalase and peroxidase (Rockey and Cello 1993). The effects of iron deficiency on piglets have not yet been fully investigated. There are some reports that document the influence of iron deficiency on the immune function (Svoboda et al. 2004) and concentrations of thyroid hormones in suckling piglets (Fajt et al. 2016).

Oxidative stress has the potential to impact the functions and survival of the organism (Yin et al. 2013). It is not yet clear whether iron deficiency can also influence the level of oxidative stress and antioxidant defenses of the organism. Studies conducted on human patients and rats offer contradictory and limited data on this issue. For instance, Kumerova et al. (1998) found an increased lipid peroxidation and decreased antioxidant defenses in human patients with iron deficiency anaemia. On the other hand, Acharya (1991) found that there was no evidence of an increased susceptibility of RBC (red blood cell count) to lipid peroxidation in iron deficiency. 
To our knowledge there are no reports dealing with this issue in piglets. Therefore, the aim of our trial was to evaluate the effects of iron deficiency on the level of oxidative stress and antioxidant defenses in suckling piglets.

\section{Experimental design}

\section{Materials and Methods}

A total of 20 piglets of Landrace $\times$ Czech Large White breed before weaning were used in the study. The piglets were weaned at the age of 28 days. They were kept under the standard conditions of farrowing units. Two different litters from two sows were used. They were fed with a standard "KPK diet" (with iron concentration of $160 \mathrm{mg} / \mathrm{kg}$ of feed) 14 days before parturition until weaning. The piglets were fed with a standard feed mixture "Weaning pellets gr" (with iron concentration of $101 \mathrm{mg} / \mathrm{kg}$ of feed) and "Starter gr" (with iron concentration of $101 \mathrm{mg} / \mathrm{kg}$ of feed). The diets were produced by De Heus a.s., Marefy, Czech Republic. We used the principle of split litters. All piglets were marked with a plastic ear tag. The piglets were weighed before each sampling. We used standard certificated and serviced laboratory scale to determine the weight of piglets. All animals used in this trial were kept in these groups until weaning and one week after. Group E was the experimental group with no iron supplementation after birth. Group $\mathrm{C}$ was the control group with iron supplementation after birth. The piglets in the control group (C) were given a single i.m. injection of iron at a dose of $200 \mathrm{mg} / \mathrm{ml}$ in the form of the gleptoferronum complex as $1 \mathrm{ml}$ of Gleptosil inj. $100 \mathrm{ml}$ (CEVA ANIMAL HEALTH Slovakia, s.r.o.) at the age of 3 days. The piglets in the experimental group (E) were given a single i.m. injection of iron at a dose of 200 $\mathrm{mg} / \mathrm{ml}$ in the form of the gleptoferronum complex as $1 \mathrm{ml}$ of Gleptosil inj. $100 \mathrm{ml}$ (CEVA ANIMAL HEALTH Slovakia, s.r.o) at the age of 21 days. Brno.

The study was approved by the Ethics Committee of the University of Veterinary and Pharmaceutical Sciences

\section{Sampling}

Blood samples were taken on days 21,28 , and 35 of age of piglets. On day 3 we did not include the indices of oxidative stress, as that would require a large amount of blood which is impossible to collect at such age.

Blood was taken from vena cava cranialis. The blood samples were left in sterile tubes with EDTA (ethylenediaminetetraacetic acid) for haematology indicators such as RBC, HGB concentration, microhaematocrit (PCV), mean corpuscular volume (MCV), mean corpuscular haemoglobin (MCH), mean corpuscular haemoglobin concentration (MCHC) and red blood cell distribution width (RDW). Special tubes with heparin were used for sampling blood for oxidative indices such as ceruloplasmin, CP (carbonyl proteins), SOD (superoxide dismutase), TEAC (trolox equivalent antioxidant capacity), TBARS (thiobarbituric acid reacting substances), GSH-Px (glutathione peroxidase) and biochemical profile and Fe.

\section{Haematology indicators analysis}

Mindway, BC - 2800 Vet. machine was used for analysis of haematology indicators in EDTA blood samples. Dia Rinse D, Dia Lyse Diff D-CF, Dia EZ Cleanser D, Probe Cleanser (Medesa, Czech Republic) were used as flush solutions. The method of colorimetry for detection of HGB was used. The method of impedance was used for other variables.

\section{Oxidative indicator analysis}

The level of lipid peroxidation in plasma was measured by quantifying the concentration of 2-thiobarbituric acid reacting substances (TBARS) with the Ohkawa et al. (1979) and with modifications by Lushchak et al. (2005) measured spectrophotometrically at $535 \mathrm{~nm}$. The TBARS concentrations were expressed as nanomoles per millilitre of plasma.

The rate of protein oxidative damage was estimated from the reaction of the resultant carbonyl derivatives of amino acid reaction with DNPH (dinitrophenylhydrasine) as described by Levine et al. (1990) and as modified by Castegna et al. (2003). Carbonyl groups were measured spectrophotometrically at $370 \mathrm{~nm}$. Concentrations of oxidatively modified proteins were expressed as nanomoles per milligram of protein.

The TEAC in the plasma was determined following Re et al. (1999) and measured spectrophotometrically at $734 \mathrm{~nm}$. The TEAC values were expressed as millimoles of trolox as external standard per millilitre of plasma.

The ceruloplasmin activity in plasma was analysed according to Ceron and Martinez-Subiela (2004) with slight modifications using the Varioskan flash spectral scanning multimode reader (Thermo Fisher Scientific Inc., USA). Results were expressed as the amount of the absorbance increase per $\min \times 10,000$.

The plasma SOD (superoxide dismutase) activity was determined as described by Beyer and Fridovich (1987). This method employs xanthine and xanthine oxidase to generate superoxide radicals, which react with 2-(4-iodophenyl)-3-(4-nitro phenol-s-phenyl tetra-zolium chloride) to form a red formazon dye. The SOD activity is then measured by the degree of inhibition of this reaction.

The activity of GSH-Px in plasma was determined spectrophotometrically and calculated from the rate of nicotinamide adenine dinucleotide phosphate-oxidase (NADPH) oxidation by the reaction with gluthatione 
reductase (GR) at $340 \mathrm{~nm}$ (Flohe and Gunzler 1984). The specific activity of was expressed as the nmol of NADPH consumption per min per mg of protein.

Biochemical indicator analysis

The plasma biochemical indices such as total protein (TP), alanine aminotransferase (ALT), aspartate aminotransferase (AST), alkaline phosphatase (ALP), lactate dehydrogenase (LDH-L), creatine kinase (CK), and creatinine (CREAT) were determined using the biochemical analyser Konelab 20i and commercial kits (Biovendor, Czech Republic).

Iron analysis

The Solaar (Thermo Electron Corporation, England) machine was used for determination of iron in serum. The method of atomic absorption spectrometry was used.

\section{Statistical analysis}

In all compared groups, normality of distribution was first tested by Shapiro-Wilks test. When the results of our groups did not meet the criteria of normality (Royston 1995), non-parametric Mann-Whitney U test was used to compare the data. Whenever the data matched the normality criteria, Student's $t$-test was used. The level of significance was accepted at $P<0.05$. The data were also tested using multicomparative test ( 2 -factor Tukey's honest significance test) to achieve comparison of the differences of mean values in all possible pairs of compared groups.

\section{Results}

\section{Oxidation factors}

The value of ceruloplasmin was significantly lower in Group E compared to Group C on day 21 . There was a significant increase in the ceruloplasmin value in Group E between days 21 and 28. In Group C, there was a significant decrease of ceruloplasmin value between days 21 and 28. Considering GSH-Px, there was no significant difference between Groups $\mathrm{C}$ and $\mathrm{E}$, at any time of the experiment. There was no significant difference in the $\mathrm{CP}$ values between groups $\mathrm{C}$ and $\mathrm{E}$ at any time of measurement. However, we noted a significant increase in the CP values between measurements on days 21 and 28 in both groups. In the TBARS measurement, we noted a significant increase in both groups $\mathrm{C}$ and $\mathrm{E}$ between days 28 and 35. The TEAC showed a significant increase in group $\mathrm{E}$ between days 28 and 35 . There was no significant difference in SOD values between group $\mathrm{C}$ and $\mathrm{E}$ at any time of experiment. The differences in oxidation factors between Group $\mathrm{E}$ and $\mathrm{C}$ are described in Table 1.

Table 1. Oxidative status of weaned piglets at different times of iron administration.

\begin{tabular}{cccccccc}
\hline $\begin{array}{c}\text { Day } \\
\text { of age }\end{array}$ & Group & $\begin{array}{c}\text { TBARS } \\
{\left[\mathrm{nmol} \cdot \mathrm{ml}^{-1}\right]}\end{array}$ & CP $\left[\mathrm{nmol} \cdot \mathrm{mg}^{-1}\right]$ & $\begin{array}{c}\text { SOD }\left[\mathrm{U} \cdot \mathrm{ml}^{-1}\right] \\
\text { NADPH }\left[\mu \mathrm{kat} \cdot l^{-1}\right]\end{array}$ & $\begin{array}{c}\text { GSH-Px } \\
{\left[\mu \mathrm{kat} \cdot \cdot^{-1}\right]}\end{array}$ & $\begin{array}{c}\text { Ceruloplasmin } \\
{\left[\mu \mathrm{M} \cdot \mathrm{ml}^{-1}\right]}\end{array}$ & TEAC \\
\hline 21 & $\mathrm{E}$ & $10.33 \pm 6.50$ & $0.29 \pm 0.08$ & $71.38 \pm 37.13$ & $12.95 \pm 1.76$ & $216.57 \pm 3.22$ & $0.96 \pm 0.07$ \\
& $\mathrm{C}$ & $8.88 \pm 6.05$ & $0.29 \pm 0.11$ & $111.64 \pm 45.94$ & $11.25 \pm 1.88$ & $376.47 \pm 124.65^{*}$ & $0.94 \pm 0.08$ \\
28 & $\mathrm{E}$ & $5.61 \pm 2.34$ & $0.66 \pm 0.20$ & $153.67 \pm 22.61$ & $13.06 \pm 1.49$ & $313.43 \pm 46.48$ & $0.88 \pm 0.22$ \\
& $\mathrm{C}$ & $7.05 \pm 3.46$ & $0.73 \pm 0.21$ & $148.21 \pm 29.21$ & $14.65 \pm 6.70$ & $279.46 \pm 54.30$ & $0.747 \pm 0.22$ \\
35 & E & $22.08 \pm 3.07$ & $0.60 \pm 0.23$ & $100.65 \pm 118.24$ & $16.62 \pm 3.00$ & $332.67 \pm 53.43$ & $1.34 \pm 0.60$ \\
& $\mathrm{C}$ & $22.50 \pm 4.16$ & $0.68 \pm 0.19$ & $67.79 \pm 81.90$ & $15.97 \pm 2.79$ & $325.17 \pm 65.51$ & $1.10 \pm 0.29$ \\
\hline
\end{tabular}

The results are expressed as mean \pm standard deviation; data are expressed as means \pm standard error of the mean. $* P<0.05$; E - experimental group; C - control group; TBARS - 2-thiobarbituric acid reacting substances; CP - carbonyl proteins, SOD - super oxid dismutase; GSH-Px - glutathione peroxidase; NADPH - nicotinamide adenine dinucleotide phosphate-oxidase; TEAC - Trolox equivalent antioxidant capacity

\section{Haematological indicators}

The RBC, HCT and HGB counts were significantly lower in Group E compared to Group $\mathrm{C}$ on days 21 and 28. There was a significant decrease in RBC, HCT and HGB values in Group E between measurements on day 3 and 21. The values of RBC, HCT, and HGB 
increased significantly between days 21 and 28 in Group E. In Group C, the RBC, HCT and HGB values increased significantly between measurements on day 3 and 21 .

There was a significantly higher value of MCV in Group C compared to Group E on day 21. There was a significant decrease in MCV values in Group E between measurements on day 3 and 21. The MCV values increased significantly between days 21 and 28 in Group E. In Group C, the MCV values decreased significantly between measurements on day 21 and 28.

There was a significantly higher value of MCHC in Group E than in Group C on day 21. There was a significant decrease in MCHC values in Group E between measurements on days 3 and 21. The values of MCHC increased significantly between days 21 and 28 in Group E.

The RDW values were significantly lower in Group C than in Group E on days 21 and 28. There was a significant increase in RDW values in Group E between measurements on day 3 and 21. The values of RDW decreased significantly between days 28 and 35 in Group E.

The differences in haematology indicators between Group $\mathrm{E}$ and $\mathrm{C}$ are described in Table 2.

Table 2. Haematology indicators and differences between the experimental (E) and control group (C).

\begin{tabular}{ccccccccc}
\hline $\begin{array}{c}\text { Day } \\
\text { of age }\end{array}$ & Group & RBC $\left(10^{\left.12 \cdot \mathrm{l}^{-1}\right)}\right.$ & $\mathrm{HGB}\left(\mathrm{g} \cdot \mathrm{l}^{-1}\right)$ & $\mathrm{PCV}(\%)$ & $\mathrm{MCV}\left(\mathrm{f} \cdot \mathrm{l}^{-1}\right)$ & $\mathrm{MCH}\left(\mathrm{p} \cdot \mathrm{g}^{-1}\right)$ & $\mathrm{MCHC}\left(\mathrm{g} \cdot \mathrm{l}^{-1}\right)$ & $\mathrm{RDW}(\%)$ \\
\hline 3 & E & $4.13 \pm 0.32$ & $68.20 \pm 6.96$ & $23.21 \pm 2.34$ & $56.26 \pm 2.89$ & $16.45 \pm 0.78$ & $293.60 \pm 11.58$ & $17.63 \pm 0.98$ \\
& $\mathrm{C}$ & $4.33 \pm 0.51$ & $70.10 \pm 6.54$ & $23.79 \pm 2.16$ & $55.25 \pm 2.90$ & $16.21 \pm 1.07$ & $294.50 \pm 12.12$ & $17.50 \pm 0.60$ \\
21 & E & $3.04 \pm 0.97$ & $41.30 \pm 5.70$ & $11.94 \pm 2.91$ & $40.34 \pm 4.28$ & $14.37 \pm 3.56$ & $354.20 \pm 54.95$ & $31.29 \pm 2.87$ \\
& C & $6.08 \pm 1.01^{*}$ & $97.20 \pm 24.27^{*}$ & $34.05 \pm 5.90^{*}$ & $56.07 \pm 3.17^{*}$ & $15.61 \pm 2.51$ & $279.00 \pm 40.30^{*}$ & $17.80 \pm 2.03^{*}$ \\
28 & E & $5.84 \pm 0.68$ & $90.80 \pm 7.38$ & $30.52 \pm 2.83$ & $52.65 \pm 4.52$ & $15.60 \pm 1.42$ & $297.40 \pm 7.93$ & $34.72 \pm 1.06$ \\
& C & $6.79 \pm 0.45^{*}$ & $101.70 \pm 13.28^{*}$ & $34.24 \pm 4.39^{*}$ & $50.41 \pm 4.93$ & $14.90 \pm 1.48$ & $296.40 \pm 7.35$ & $17.98 \pm 3.67 *$ \\
35 & E & $6.60 \pm 0.52$ & $93.00 \pm 6.00$ & $33.21 \pm 2.46$ & $50.54 \pm 3.42$ & $14.10 \pm 1.16$ & $280.00 \pm 13.61$ & $19.48 \pm 4.77$ \\
& C & $7.08 \pm 0.40$ & $99.50 \pm 11.54$ & $35.43 \pm 4.00$ & $50.07 \pm 4.53$ & $13.98 \pm 1.38$ & $280.30 \pm 8.29$ & $17.59 \pm 4.06$ \\
\hline
\end{tabular}

The results are expressed as mean \pm standard deviation; data are expressed as means \pm standard error of the mean. $* P<0.05$; E - experimental group; C - control group; RBC - red blood cells; HGB - haemoglobin concentration; PCV - microhaematokrit; MCV - mean corpuscular volume; $\mathrm{MCH}$ - mean corpuscular haemoglobin; MCHC - mean corpuscular haemoglobin concentration; RDW - red blood cell distribution width

\section{Biochemical indicators}

With regard to the lactate count, Group E showed significantly higher values on day 21 compared to Group C.

In the Fe count, Group E showed significantly lower values on day 21 compared to Group C. There was a significant increase in Fe values in Group E between measurements on days 21 and 28. The differences in iron plasma concentration between Group E and C are described in Table 3.

Weight of piglets

The weights of the piglets showed no significant difference between the experimental group (Group E) and the control group (Group C) at any time of the experiment.

\section{Discussion}

As expected, iron deficiency in our experimental group resulted in the development of anaemia. Bhattarai and Nielsen (2015) consider a haemoglobin level below $90 \mathrm{~g} / \mathrm{l}$ as the definition of anaemia. In our study the values were significantly lower than this 


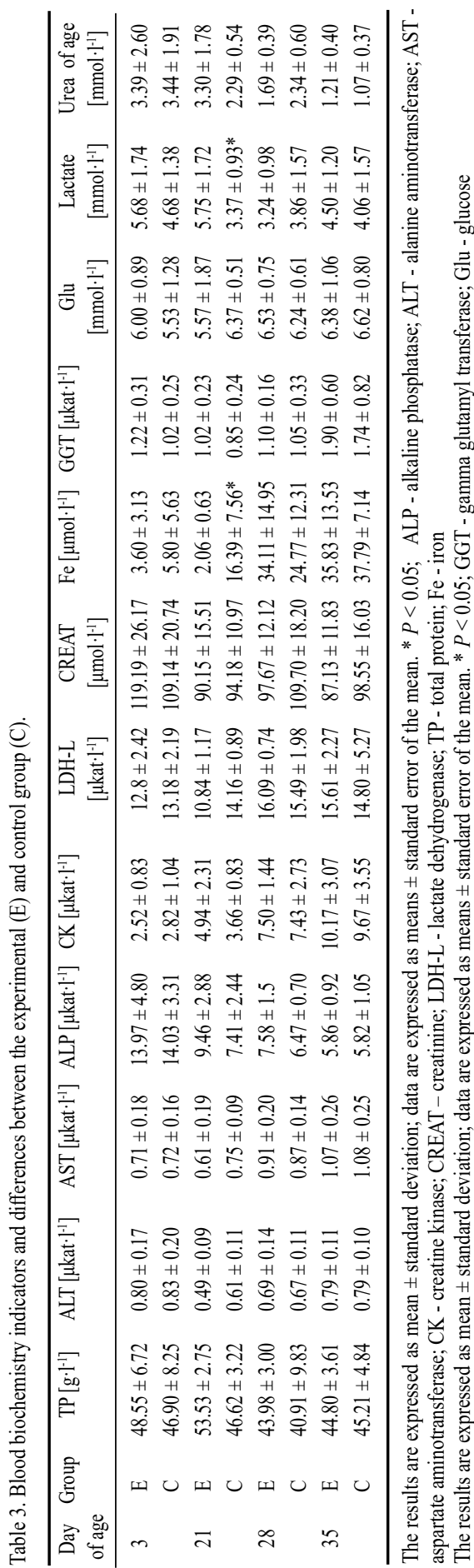

limit. The other haematological indices and iron concentration in blood plasma were also significantly lower in the experimental group compared to control.

Oxidative stress has been defined as a disturbance of equilibrium between the production of reactive oxygen species and the antioxidant defence system ( $\mathrm{Pi}$ et al. 2010) including superoxide dismutase (SOD), catalase, and glutathione peroxidase (GSH-Px) (Han et al. 2011). Increased lipid peroxidation has been associated with iron deficiency in rats and human patients (Kumerova et al. 1998), but the topic remains controversial (Acharya et al. 1991). In case of the iron deficiency in suckling piglets diagnosed in our trial, changes in the following indices of oxidative status can be discussed.

\section{TBARS}

TBARS are secondary products of lipoperoxidation (Tsaknis et al. 1998). No significant difference between anaemic and iron supplemented piglets were found in any period of the study. This indicates that the level of lipid peroxidation was not affected by iron deficiency. One week after weaning there was a significant increase in TABRS levels in both groups. This is in line with Zhu et al. (2012) and Yin et al. (2014) and indicates that weaning was associated with increased oxidative stress.

\section{Carbonyl proteins}

Carbonyl proteins are products of oxidized proteins (Dalle et al. 2003). No significant differences between anaemic and control groups were found during the study. This indicates that the protein oxidation was not influenced by iron deficiency.

\section{Super oxide dismutase}

According to the results obtained by Kumerova et al. (1998) and Isler et al. (2002) the SOD activity was significantly lower in anaemic human patients. This indicates that the antioxidant defence is decreased in anaemic patients. In our study we also found a lower mean value of SOD activity in anaemic piglets, however, the 
difference was not significant. One week after weaning the variability of this indicator significantly increased. This may be caused by different degrees of stress in individual piglets.

\section{Glutathione peroxidase}

GSH-Px activities in anaemic piglets were similar to those of the control group. This is in agreement with Isler et al. (2002) and indicates that the enzyme was not affected by iron deficiency.

\section{Ceruloplasmin}

Ceruloplasmin has an important role in iron metabolism. It plays a major role in oxidizing $\mathrm{Fe}^{2+}$ to $\mathrm{Fe}^{3+}$. It therefore converts the toxic ferrous form to its non-toxic ferric form which is associated with transferrin (De Silva and Aust 1992). An increased activity of ceruloplasmin was found in iron deficient rats (Ranganathan et al. 2011). This could be a compensatory response to maintain iron homeostasis under conditions of its deficiency. In contrast, in the anaemic suckling piglets we have significantly lower ceruloplasmin activities compared to the control group. More studies are necessary to investigate the topic and clarify the differences.

\section{Total antioxidant capacity}

There were no significant differences in the total antioxidant capacity of plasma between the anaemic piglets and the control. This is contradictory to Aslan et al. (2006) who found that the total antioxidant capacity was significantly lower in anaemic human patients. More research is necessary to further investigate this problem and explain the differences.

One week after weaning, TEAC increased significantly in the experimental group. This could be attributed to the weaning stress.

It can be concluded that the levels of lipid peroxidation and protein oxidation were not affected in anaemic suckling piglets. The antioxidant defence (ceruloplasmin) was influenced only partially by iron deficiency.

\section{Acknowledgements}

This research was supported by the Internal Grant Agency VFU Brno 111/2016/FVL, University of Veterinary and Pharmaceutical Sciences Brno, Czech Republic.

\section{References}

Acharya J, Punchard NA, Taylor JA, Thompson RP, Pearson TC 1991: Red cell lipid peroxidation and antioxidant enzymes in iron deficiency. Eur J Haematol 47: 287-291

Aslan M, Horoz M, Kocyigit A, Ozgonül S, Celik H, Celik M, Erel O 2006: Lymphocyte DNA damage and oxidative stress patients with iron deficiency anemia. Mutat Res 601: 144-149

Beyer WF, Fridovich I 1987: Assaying for superoxide dismutase activity: some large consequences of minor changes in conditions. Anal Biochem 161: 559-566

Bhattarai S, Nielsen JP 2015: Early indicators of iron deficiency in large piglets at weaning. J Swine Health Prod J 23: $10-17$

Castegna A, Drake J, Pocernich C, Butterfield DA 2003: Protein carbonyl levels-an assesment of protein oxidation. In: Methods in Biological Oxidative Stress (Methods in Pharmacology and Toxicology), Human Press, pp 161-168

Ceron JJ, Martinez-Subiela S 2004: An automated spectrophotometric method for measuring canine ceruloplasmin in serum. Vet Res 35: 671-679

Dalle-Donne I, Rossi R, Giustarini D, Milzani A, Colombo R 2003: Protein carbonyl groups as biomarkers of oxidative stress. Clin Chim Acta 329: 23-38

De Silva DM, Aust SD 1992: Ferritin and ceruloplasmin in oxidative damage: review and recent findings. Can J Physiol Pharmacol 71: 715-720

Fajt Z, Baňoch T, Vašek J, Vaňhara J, Blahová J, Kazatelová Z, Fiorino E, Svoboda M 2016: Influence of iron deficiency on thyroidal hormone production in piglets before weaning. Act Vet Brno 85: 17-22 
Flohe L, Gunzler WA 1984: Assays of glutathione peroxidase. Met Enz 105: 114-121

Han J, Shuvaev VV, Muzykantov VR 2011: Catalase and SOD conjugated with PECAM antibody distinctly alleviate abnormal endothelial permeability caused by exogenous ROS and vascular endothelial growth factor. J Pharmacol Exp Ther 338: 82-91

Isler M, Delibas N, Guclu M, Gultekin F, Sutcu R, Bahceci M, Kosar A 2002: Superoxide dismutase and glutathione peroxidase in erythrocytes of patients with iron deficiency anemia: Effects of different treatment modalities. Croat Med J 43: 16-19

Kumerova A, Lece A, Skesters A, Silova A, Petuhovs V 1998: Anaemia and antioxidant defence of the red blood cells. Mater Med Pol 30: 12-15

Levine R, Garland D, Oliver CN 1990: Determination of carbonyl content in oxidatively modified proteins. Met in Enzym 186: 464-478

Lushchak VI, Bagnyukova TV, Lushchak OV, Storey JM, Storey KB 2005: Hypoxia and recovery perturb free radical processes and antioxidant potential in common carp (Cyprinus carpio) tissues. Int J Biochem Cell Biol 37: $1319-1330$

Nagababu E, Gulyani S, Early ChJ, Cutler AG, Mattson MP, Rifkind JM 2008: Iron-deficiency anemia enhances red blood cell oxidative stress. Free Radic Res 42: 824-829

Ohkawa H, Ohishi N, Yagi K 1979: Assay for lipid peroxides in animal-tissues by thiobarbituric acid reaction. Anal Biochem 95: 351-358

Pi J, Zhang Q, Fu J, Woods CG, Hou Y, Corkey BE, et al. 2010 ROS signaling, oxidative stress and Nrf2 in pancreatic beta-cell function. Toxicol Appl Pharmacol 244: 77-83

Ranganathan PN, Lu Y, Jiang L, Kim Ch, Collins JF 2011: Serum ceruloplasmin protein expression and activity increases in iron-deficient rats and is further enhanced by higher dietary copper intake. Blood 118: $3146-3153$

Re R, Pellegrini N, Proteggente A, Pannala A, Yang M, Rice-Evans C 1999: Antioxidant activity applying an improved ABTS radical cation decolorization assay. Free Radic Biol Med 26: 1231-1237

Rockey DC, Cello JP 1993: Evaluation of the gastrointestinal tract in patients with iron deficiency anemia. N Engl J Med 329: 1691-1695

Royston P 1995: A remark on Algorithm AS 181: The W-test for normality. Appl Stat 44: 547-551

Svoboda M, Drábek J, Krejčí J, Reháková Z, Faldyna M 2004: Impairment of the peripheral lymphoid compartment in iron-deficient piglets. J Vet Med 51: 231-237

Tsaknis J, Stavros L, Michael H, Gillian S, Vassiliki T 1998: Rapid high-performance liquid chromatographic method of determining malondialdehyde for evaluation of rancidity in edible oils. Analyst 123: $325-327$

Venn JAJ, McCance RA, Widdowson EM 1947: Iron metabolism in piglet anaemia. J Comp Pathol Ther 57: 314-325

Yin J, Wu MM, Xiao H, Ren WK, Duan JL, Yang G, Li TJ, Yin YL 2014: Development of an antioxidant system after early weaning in piglets. J Animal Sci 92: 612-619

Yin J, Ren WK, Wu XS, Yang G, Wang J, Li TJ, Ding JN, Cai LC, Su DD 2013: Oxidative stress-mediated signaling pathways: A review. J Food Agric Environ 11: 132-139

Zhu LH, Zhao KL, Chen XL, Xu JX 2012: Impact of weaning and an antioxidant blend on intestinal barrier function and antioxidant status in pigs. J Animal Sci 90: 2581-2589 